of the professors of mathematics here with whom I have com? in contact have taken the trouble of solving it, and therefore it is that $\mathrm{I}$ am appealing in this strain to the math smaticians who have any interest in the chronolozy of India.

Many interested scholars have expressed their desire for the solution of this problem. Thus says Prof. Schle yel: "I leave to astronomers to examine whether the parts of the description agree with one another, and if this be the case, thence to deduce the date. The Indians place the nativity of $\mathrm{R} i \mathrm{~m} \mathrm{r}$ in the confines of the second age (Treta) and the third (D vapara); but it seems that this should be taken in an alle grorical sen ie. .... We may consider that the poet had an eye to the tima in which, imnediately before his own age, the aspects of the heavenly bodies were such as he has described."

Besides the positions of the planets at the birth of Rama, I have a few more data concerning the Hindu system of the division of heaven;, which I shall be glad to communicate to any gentleman who is acquainted with that system, and expresses a desire for the same.

Lahore, August 8.

KANHAIYALAL.

\section{Arrangements for Work of Chemical Section of the British Association.}

MANY of your readers will be interested to know that M. Moisian has kindly arranged for a demonstration of the properties of fluorine, and his method of isolating the element, at a meeting of the Chemical Ssction of the British Association at Nottingham, in September. M. Moissan will also exhibit some specimens of the diamonds he has artifically produced.

We also hope to have at least two discussions, one on Bacteriology and its related chemical problem, which Prof. Percy Frankland has been so good as to promise to open, and another on explosions in coal mines, with special reference to the "dust theory." This, Piof. Harold Dixon has kindly consented to introduce.

Permit me to remind authors that they will greatly facilitate the arrangements for the satisfactory grouping of papers at the sectional meeting, if they will communicate as early as possible with the Secretaries, Burlington House.

$$
\text { J. EMerson Reynolds. }
$$

University of Dublin, Trinity College, August 2 I.

\section{The Bacchus Marsh Boulder Beds.}

YoUR issue of August ro contains an interesting communication by Messrs. Officer and Balfour on the glacial boulder beds of Bacchus Marsh in Victoria, in which they are referred to as triassic. May I be permttted to point out that this is erroneous? It is true that the late Dr. O. Feistmantel in his earlier descriptions of, and references to, the flora of these beds, regarded them as triassic, but this was the natural consequence of their correlation with the Talchir group of India, which he then ascribed to the trias. In 1886 it was shown by Dr. Wagner and myself sumultaneously that the true correlation of the Talchir group was with the marine beds below the Newcastle coal measures of New South Wales; the Bacchus Marsh boulder beds are consequently upper carboniferous, and form part of the traces of the upper carboniferous glacial period which have been recognised in Australia, Africa and Asia. The matter will be found fully dealt with in pages 12O-I23 and I9I-2I4 of the second edition of the "Manual of the Geology of India," just published by the Indian Geological Survey.

S.S. Rena, August 19.

R. D. Oldham.

\section{Old and New Astronomy.}

WHILE thanking you and your reviewer for your very courteous and appreciative notice of the "Old and New Astronomy," I should like to be permitted to point out that the chapter on the sun's surroundings was printed and was in the hands of the public befure my connection with the work commenced. Your reviewer suggests that I ought to have corrected certain statements in this earlier part of the volume, but I felt that it was sufficient to mention in the preface that I did not agree with all Mr. Proctor's conclusions, and it must be obvious that it would neither have been an easy nor a gracious ta $\mathrm{k}$ to have attempted in the latter part of a book to criticise the statements and theories of the deceased author of the earlier chapters.
Your reviewer suggests that the proof I give of the very small density of the Orion Nebula is " vitiated by the fact that it is impossible to estimate the gravitational effect of the dark matter in interstellar space." I conclude that he does not refer to dark matter within the area of the nebula, for this would only add to the mass of the nebula and to the observed velocities of the stars in its neighbourhood. That dark matter evenly distributed in space around would not interfere with the gravitational effect of the nebula will be evident to those who remember that the attraction of a hollow spherical shell on a body within it may be neglected, since the attractions in all directions balance whatever is the position of the body within the spherical shell. The stars around the nebula seem to be distributed in al directions, and it cannot be supposed that in each case there is a gravitating dark mass on the side away from the nebula which counterbalances the attraction of nebulous matter.

Your reviewer cannot, I think, have seen Dr. Huggins's photo. graph of the spectrum of the Orion Nebula and the stars in volved in it, or he would not suggest that the bright lines in the spectra of the stars could be due to the overlapping by tremors and atmospheric disturbances of the spectrum of the nebular matter. The bright nebula lines distinctly widen and brighten in the neighbourhood of the stars. One is therefore forced to conclude that the bright lines when they cross the stellar spectra belong to the stars.

August 19.

THERE is nothing in my review that implies the desirability of Mr. Ranyard having modified any of Mr. Proctor's conclu. sions in the chapter on the sun's surroundings ; I simply pointed out an error of date that should have been corrected.

I do not see any reason for supposing that the quantity of matter in the area we call the Orion Nebula is greater than that in any other arbitrarily selected equal area in space, and the evidence seems conclusive that the whole of interstellar space is a meteoric plenum. This being so, there is no reason why stars in the neighbourhood of the nebula should have their velocities in any way affected by it. Mr. Ranyard proves that there is no great difference in density, but does not touch the question of the absolute quantity of matter involved.

I have not seen Dr. Huggins's original photographs of the spectrum of the Orion Nebula, and the stars involved, nor have I seen direct copies of them, but I have examined other photographs in which the star and nebula spectra appear, and have also visually examined the spectra, and am by no means convinced that the nebula lines are brighter and wider in the stars.

THE REVIEWER.

\section{An Old Device Resuscitated.}

IN the Astronomical Column of the current number of NATURE a description is given from L'Astronomie, of M. Jarson's method of giving equatorial motion to a telescope mounted on an ordinary altazimuth stand. This method will be found figured and described by Lord Lindsay, now Earl Crawford, in the Monthly Notices of the Royal Astronomical Society, xxxvii. I, Nov, 10, 1876 . Moreover, in a note appended to the paper, Lord Lindsay says that since writing it his attention had been drawn to the fact that this principle of mounting had been described by Sir George Airy in Monthly Notices, xv.

F. W. LEVANDER.

30, North Villas, Camden Square, N.W., August 28.

\section{Laws of Error in Drawing.}

I SHOULD be obliged if you allow me to state in the next issue of NATURE, to prevent any misconception of the extent of those "compulsory errors" alluded to in my article printed in the current number, that I am able to specify such, with equal particularity, as they occur in the following additional figures, viz. :-The cylinder, the ringed cylinder, the oblong, the octagon, cone, hexagonal pyramid, octagonal pyramid, triangle (solid). Also in those figures framed of square-sectioned woodwork that have the following shapes, viz. square, cross, triangle, arch, hexagon, pentagon, circle. Again, that they are observable in all forms which are complexities of the primal forms above given; and, moreover, I have traced them clearly in the draughtsmanship of Orientals, and even in the drawing of the greatest painters. ARTHUR L. HADDON.

Glenavon, Cornwallis Avenue, Clifton Vale, Bristol, August 25. 